\title{
Outcome after aortic, axillary, or femoral cannulation for acute type $A$ aortic dissection
}

\author{
Maximilian Kreibich, MD, ${ }^{\mathrm{a}, \mathrm{b}}$ Zehang Chen, BA, ${ }^{\mathrm{a}}$ Bartosz Rylski, MD, ${ }^{\mathrm{b}}$ Joseph E. Bavaria, MD, ${ }^{\mathrm{a}}$

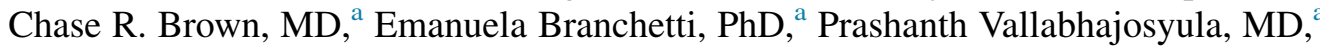 \\ Wilson Y. Szeto, MD, ${ }^{a}$ and Nimesh D. Desai, MD, $\mathrm{PhD}^{\mathrm{a}}$
}

\section{ABSTRACT}

Objective: The optimal method for arterial cannulation in acute aortic dissection type A (ADA) remains controversial. The aim of this study was to compare central ascending aortic, axillary, and femoral cannulation in patients who underwent surgery for acute ADA.

Methods: Between 2006 and 2017, 584 patients were operated on for acute ADA. Of those, $355(61 \%)$ underwent ascending aortic, $101(17 \%)$ right axillary, and $128(22 \%)$ femoral cannulation for arterial inflow. Clinical features and outcomes were compared after inverse probability weighting.

Results: After inverse probability weighting there were no statistical differences in preoperative characteristics. Operative details differed significantly among the 3 groups: hemiarch replacement was performed more often in the central aortic and the femoral group $(P<.001)$, whereas total arch replacement was performed more often in the axillary group $(P<.001)$. Cardiopulmonary bypass $(P=.022)$ and aortic cross-clamp $(P=.021)$ times were shortest in the aortic cannulation group and longest in the femoral cannulation group. Postoperative morbidities were similar; procedure-related stroke $(P=.783)$ and the need for renal replacement therapy $(P=.446)$. In-hospital mortality $(P=.680)$ and long-term survival were similar (log rank, $P=.704)$. Multilevel multivariate mixed effect logistic regression showed that the cannulation strategy was not associated with in-hospital mortality.

Conclusions: Central ascending aortic cannulation in patients with ADA can be used as safely as axillary or femoral cannulation, providing another option for quick and easy establishment of cardiopulmonary bypass. ( $\mathrm{J}$ Thorac Cardiovasc Surg 2019;158:27-34)

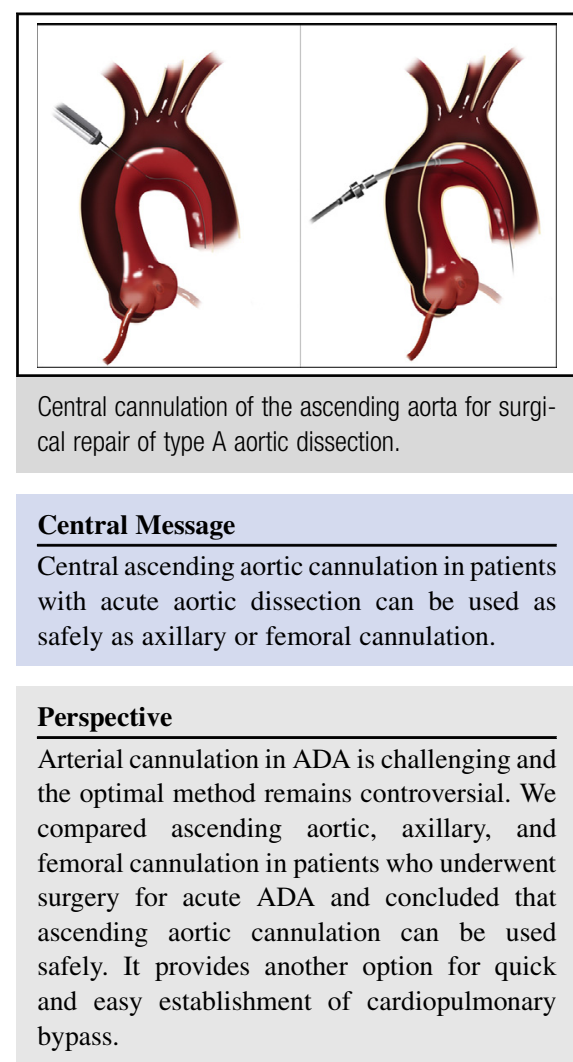

See Commentaries on pages 35 and 37.
Arterial cannulation for type A aortic dissection (ADA) has traditionally been achieved through the common femoral artery or the right axillary artery, and both are the most commonly used cannulation strategies in current practice. ${ }^{1-3}$ Direct aortic cannulation has also been suggested

\footnotetext{
From the a Division of Cardiovascular Surgery, Hospital of the University of Pennsylvania, Philadelphia, Pa; and ${ }^{b}$ Department of Cardiovascular Surgery, Heart Center University Freiburg, Faculty of Medicine, University of Freiburg, Freiburg, Germany.

Support for this study was provided by institutional funds

Received for publication Feb 7, 2018; revisions received Nov 19, 2018; accepted for publication Nov 25, 2018.

Address for reprints: Nimesh D. Desai, MD, PhD, Division of Cardiovascular Surgery, Hospital of the University of Pennsylvania, 6 Silverstein, 3400 Spruce St, Philadelphia, PA 19104 (E-mail: nimesh.desai@uphs.upenn.edu). $0022-5223 / \$ 36.00$

Copyright (C) 2018 Published by Elsevier Inc. on behalf of The American Association for Thoracic Surgery

https://doi.org/10.1016/j.jtcvs.2018.11.100
}

to be another effective cannulation option by various groups. ${ }^{4-6}$ However, the optimal cannulation site remains controversial and a general consensus is missing because of a lack of sufficient data. ${ }^{1-3}$ In fact, various studies have compared central aortic or axillary cannulation with femoral cannulation and showed no statistical difference or were in favor of nonfemoral cannulation. ${ }^{5,7}$ Also, there is only 1 recent study with a relatively small population that compared axillary and aortic cannulation, indicating comparable early results but poorer long-term outcome in the central aortic cannulation group.

Scanning this QR code will
take you to the article title
$\begin{aligned} & \text { page to access supplementary } \\ & \text { information. }\end{aligned}$




\section{Abbreviations and Acronyms \\ ADA $=$ aortic dissection type A \\ $\mathrm{CPB}=$ cardiopulmonary bypass \\ $\mathrm{CT}=$ computed tomography \\ $\mathrm{HCA}=$ hypothermic circulatory arrest \\ $\mathrm{RCP}=$ retrograde cerebral perfusion \\ TEE $=$ transesophageal echocardiography}

Our group has used central aortic cannulation, axillary cannulation, and femoral cannulation for arterial cardiopulmonary bypass $(\mathrm{CPB})$ inflow in ADA patients for more than 10 years. The aim of this study was to evaluate early and late outcome of the 3 different cannulation methods. To reduce the effect of selection bias and potential confounding, this investigation was performed after inverse probability weighting.

\section{METHODS \\ Patients}

The study population consisted of 584 patients operated on for acute ADA between January 2006 and June 2017. Diagnosis was confirmed using computed tomography (CT) scan and intraoperative transesophageal echocardiography (TEE). Patients were divided into 3 groups according to the cannulation site: central ascending aortic cannulation, right axillary cannulation, and femoral cannulation. Patients with previous cardiac surgery were not included in the study. We compared clinical features and outcomes after surgery. In addition, a subgroup analysis was performed for the safety of axillary cannulation in patients with the dissection involving the innominate and right axillary artery. The institutional review committee approved this retrospective study and the need for informed consent was waived.

\section{Outcome Measures}

Outcomes included postoperative, 1-month, and late follow-up data obtained from our center's aortic database that includes all available data from the University of Pennsylvania Health System as well as information gathered from patients' general practitioners or the patients and their family members. All patients were routinely followed-up after 1 month, and patients were encouraged to present to the aortic clinic for late follow-up. In case follow-up data were older than 6 months in surviving patients the University of Pennsylvania Health System database was screened for any entry. In patients without an entry, social security death index was screened for additional information. Follow-up data were available in 516 of 518 hospital survivors $(99.6 \%)$. Patients were followed-up for a total of 2100 patient-years, with a median follow-up among survivors of $4.1 \pm 3.1$ years. Organ malperfusion was diagnosed with clinical examination and confirmed using CT angiography and was defined as altered blood flow with clinical evidence of a lack of blood flow resulting in ischemia with organ dysfunction. Shock was defined as persistent hypotension (systolic blood pressure $<80 \mathrm{~mm} \mathrm{Hg}$ ) with severe reduction in cardiac index. Dissection-related stroke was defined as postoperative stroke in patients with clear preoperative neurologic deficit in the absence of hemodynamic compromise, whereas procedure-related stroke was defined as postoperative stroke in patients without clear preoperative neurologic deficit in the absence of hemodynamic compromise. Overall stroke included all patients with either dissection-related or procedure-related stroke. Early mortality was defined as in-hospital death and length of in-hospital stay was censored if a patient died during the hospital stay. Data for clinical presentation at admission and initial extent of the dissection were gathered from preoperative documentation.

\section{Surgical Approach}

For all patients a previously described standardized integrated surgical management was applied. This approach includes fast-track direct admission to the operating room and immediate surgical repair. ${ }^{9,10}$ The proximal aortic repair included the replacement of the entire ascending aorta either with resuspension of the aortic valve (supracoronary ascending replacement, sinus of Valsalva repair with a Teflon felt neo-media and valve resuspension in patients with native aortic valve), aortic root replacement with a valved conduit, a Wheat procedure (aortic valve replacement, sinus of Valsalva repair with a Teflon felt neo-media and supracoronary ascending replacement), or a valve-sparing aortic root replacement as described previously. ${ }^{9,10}$ The distal repair included isolated ascending, hemiarch, or complete arch replacement depending on the entry tear location and aortic diameter, as described previously. ${ }^{11,12}$ In short, the entire residual ascending aorta and most of the lesser curvature of the aortic arch were routinely replaced in an aggressive, extended transverse hemiarch fashion and dissected layers were repaired with Teflon felt neo-media. Isolated ascending replacement was performed only in rare instances ( 7 patients in the study period). In case of an entry tear at the larger curvature of the aortic arch, complete arch replacement was performed. Aortic arch surgery was performed with the patient in hypothermic circulatory arrest (HCA) with selective cerebral perfusion and open distal anastomosis in all but 5 patients in the study. In those 5 patients, the aortic cross-clamp was placed distally enough for an isolated ascending replacement with complete removal of dissected tissue. Cerebral protection was achieved by either antegrade cerebral perfusion or retrograde cerebral perfusion (RCP). In those requiring more extensive arch reconstruction necessitating more than 40 minutes of circulatory arrest and RCP, selective unilateral or bilateral antegrade cerebral perfusion was typically done using balloon-tipped cannulas placed in the innominate and left common carotid arteries. In patients with right axillary cannulation, selective antegrade cerebral perfusion was established through the axillary artery by clamping the innominate artery and, when needed, a second balloon-tipped cannula placed in the left common carotid artery. In case of femoral or central aortic cannulation, an arterial cannula was inserted in the graft and antegrade CPB was restarted after HCA.

Specific technical details on ascending aortic cannulation have been previously described by our group (Video 1 ). ${ }^{6}$ Briefly, the relationship of the true and false lumen in the ascending aorta and aortic arch were evaluated according to CT angiography, intraoperative TEE, and during visual examination of the ascending aorta. The true lumen was cannulated using

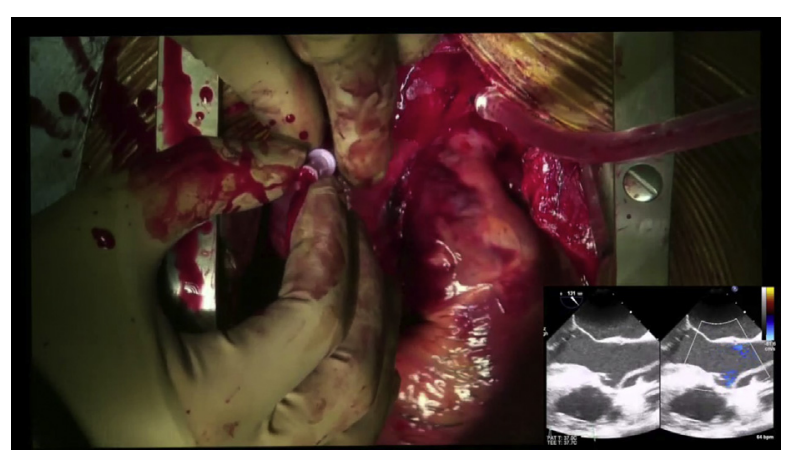

VIDEO 1. Central ascending aortic cannulation in a patient presenting with acute type A aortic dissection. Video available at: https://www. jtcvs.org/article/S0022-5223(18)33248-3/fulltext. 


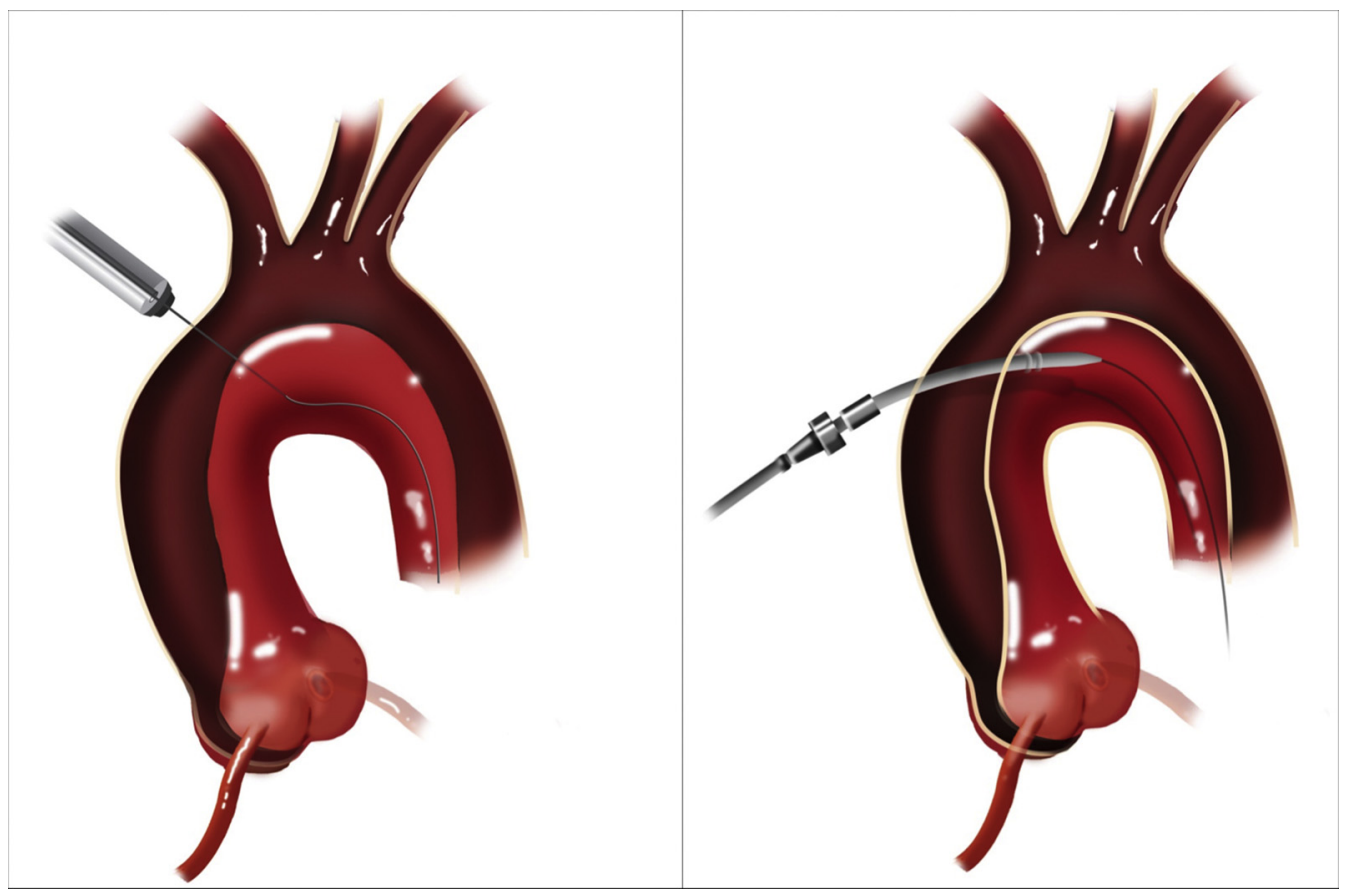

FIGURE 1. Central cannulation of the ascending aorta for surgical repair of type A aortic dissection. The relationship of the true and false lumens in the ascending aorta and aortic arch are evaluated according to computed tomography angiography, intraoperative transesophageal echocardiography, and during visual examination of the ascending aorta. The true lumen is cannulated using the Seldinger technique and correct wire and cannula placement in the true lumen is confirmed using transesophageal echocardiography.

the Seldinger technique (Figures 1 and 2). Careful collaboration with the echocardiographer is of paramount importance, and the wire has to be well down in the true lumen within the descending aorta before dilatation. In patients with large arch tears, we are especially careful, because it might be difficult to keep the wire in the true lumen when advancing the wire distally. Femoral cannulation was performed in a similar fashion with true lumen cannulation using the Seldinger technique and TEE confirmation of correct wire placement. Axillary cannulation was performed through a Dacron graft anastomosed to the axillary artery. The cannulation sites were selected primarily according to the surgeons' preference and the location of the primary entry tear.

\section{Statistical Analysis}

We used inverse probability weighting to limit confounding by indication (see the Appendix E1 for details). Weights were calculated using the procedures implemented in the twang package for R. ${ }^{13,14}$ Because no significant outliers were observed in weights among cannulation groups, they were used for the regressions. Balance between cannulation groups was assessed with the use of standardized mean differences. A standardized difference of $\leq 10 \%$ was considered an ideal balance, and a standardized difference of $\leq 30 \%$ was considered an acceptable balance. Bias reduction for variables with $>20 \%$ standardized difference are reported in Table E1 as well.

Data are presented as mean \pm standard deviation for normally distributed variables and as median (first quartile-third quartile) for nonparametrically distributed variables. Analysis of variance or the Kruskal-Wallis test were used for comparisons of continuous variables. Categorical variables were compared using the $\chi^{2}$ test with the calculation of exact values and are shown as number (percentage). In case of small group sizes $(\mathrm{n}<5)$, the Fisher exact test was used.

A weighted 2-level multivariate mixed effect logistic regression with a robust variance estimator was used to determine the marginal effect of different cannulation groups on in-hospital mortality as well as the influences of selected variables (sex, chronic obstructive pulmonary disease, chronic renal failure, age $>80$ years, shock, tamponade, any organ malperfusion, and cannulation type), with surgeon-specific factors included as a random effect. Weighted Cox regression with a robust variance estimator was used to estimate long-term mortality differences between cannulation groups. As sensitivity analyses, stabilized weights were also generated and applied to the aforementioned logistic and Cox models (see the Appendix E1 for details). ${ }^{15}$ All tests were performed with an $\alpha$ threshold of .05. The statistical analysis was performed using STATA version 15.1 (STATA Corp, College Station, Tex) and R version 3.5.1 (R Foundation for Statistical Computing, Vienna, Austria).

\section{RESULTS}

\section{Patient Characteristics}

Of the 584 unweighted patients, central aortic cannulation was performed in $355(61 \%)$ patients, right axillary cannulation was performed in $101(17 \%)$ patients, and femoral cannulation was performed in 128 (22\%) patients. After inverse probability weighting, there were no difference in baseline patient characteristics (Table E2). Characteristics before inverse probability weighting are summarized in Table 1.

\section{Clinical Presentation}

Before inverse probability weighting, significantly more patients presented in shock in the aortic and the femoral cannulation group and significantly more patients in the 


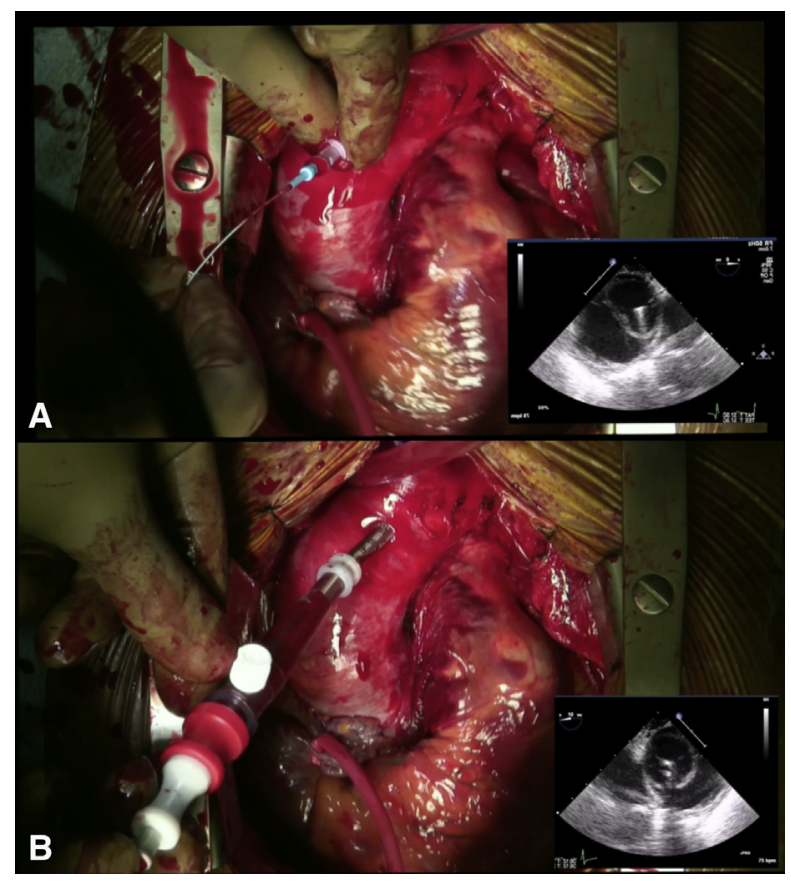

FIGURE 2. Intraoperative view. The distal ascending aorta is cannulated using the Seldinger technique and correct wire (A) and cannula placement (B) in the true lumen is confirmed using transesophageal echocardiography.

axillary and femoral group had complete aortic dissection compared with the central aortic cannulation group (Table 2). There was no difference after inverse probability weighting (Table E3).

\section{Operative Details}

Of the 584 surgeries for ADA, 548 surgeries were performed by 1 of the 10 aortic surgeons working at the University of Pennsylvania Health System during the study period. Thirty-six surgeries were performed by other cardiothoracic surgeons performing $<20$ ADA surgeries a year. Operative details and times are summarized in Table 3. Although there was no difference in the proximal aortic repair (aortic valve resuspension was the most frequent proximal repair in all groups), the distal aortic repair differed significantly among the 3 groups: hemiarch replacement was performed more often in the aortic and the femoral cannulation groups (more than $90 \%$ of all patients), whereas total arch replacement was performed significantly more often in the axillary group. Operative times were shortest in the aortic cannulation group. RCP was the preferred method of cerebral perfusion in the aortic and the femoral cannulation group, whereas antegrade cerebral perfusion was significantly more common in the axillary cannulation group.

\section{Outcome Characteristics}

The distribution of perioperative complications was similar among all groups (Table 4): there was no statistical difference in the tracheotomy rate, the rate for re-exploration for bleeding, procedure-related and/or dissection-related stroke, temporary neurological dysfunction, renal failure, or the need for renal replacement therapy. The length of stay in the intensive care unit as well as the time to discharge were similar among the 3 groups. In-hospital mortality (aortic: 10\% vs axillary: $11 \%$ vs femoral: $13 \% ; P=.680$ ) was comparable among the groups and was lowest in the aortic cannulation group. Long-term survival did not differ (Figure 3; log rank, $P=.704)$.

\section{Logistic Regression Analysis}

The multilevel multivariate mixed effect logistic regression showed that the cannulation strategy was not associated with in-hospital mortality, after adjusting for surgeon-specific factors (Table 5). However, age $>80$ years (odds ratio, 5.000; $P=.018$ ) and any organ

TABLE 1. Baseline characteristics of the study population before inverse probability weighting

\begin{tabular}{|c|c|c|c|c|}
\hline & \multicolumn{3}{|c|}{ Cannulation group } & \multirow[b]{2}{*}{$P$ value } \\
\hline & $\overline{\operatorname{Aortic}(n=355)}$ & Axillary $(n=101)$ & Femoral $(n=128)$ & \\
\hline Age, y & $60 \pm 14$ & $58 \pm 14$ & $60 \pm 14$ & .443 \\
\hline Male sex & $222(63)$ & $70(69)$ & $83(65)$ & .449 \\
\hline Hyperlipidemia & $137(39)$ & $48(48)$ & $56(44)$ & .184 \\
\hline Hypertension & $315(89)$ & $90(89)$ & $112(88)$ & .964 \\
\hline Diabetes mellitus type 2 & $47(13)$ & $12(12)$ & $18(14)$ & .896 \\
\hline Previous stroke & $31(9)$ & $9(9)$ & $14(11)$ & .844 \\
\hline Chronic renal failure & $35(10)$ & $17(17)$ & $17(13)$ & .145 \\
\hline COPD & $46(13)$ & $13(13)$ & $16(13)$ & .969 \\
\hline Coronary artery disease & $35(10)$ & $6(6)$ & $16(13)$ & .281 \\
\hline Bicuspid aortic valve & $9(3)$ & $9(9)$ & $4(3)$ & .020 \\
\hline Marfan syndrome & $5(1)$ & $5(5)$ & $3(2)$ & .091 \\
\hline
\end{tabular}

Values are $\mathrm{n}(\%)$ or mean \pm standard deviation. Bold indicates significant results $(P<.05)$. COPD, Chronic obstructive pulmonary disease. 
TABLE 2. Clinical characteristics of the study population before inverse probability weighting

\begin{tabular}{|c|c|c|c|c|}
\hline & \multicolumn{3}{|c|}{ Cannulation group } & \multirow[b]{2}{*}{$P$ value } \\
\hline & $\overline{\text { Aortic }(n=355)}$ & Axillary $(n=101)$ & Femoral $(n=128)$ & \\
\hline Shock & $107(30)$ & $17(17)$ & $39(30)$ & .026 \\
\hline Tamponade & $79(22)$ & $18(18)$ & $33(26)$ & .380 \\
\hline Moderate to severe AR & $116(33)$ & $27(27)$ & $49(38)$ & .222 \\
\hline Any organ malperfusion & $134(38)$ & $33(33)$ & $49(38)$ & .606 \\
\hline \multicolumn{5}{|l|}{ Dissection extension } \\
\hline Limited, proximal dissection & $105(30)$ & $16(16)$ & $23(18)$ & .026 \\
\hline Supra-aortic vessel dissection & $143(40)$ & $46(46)$ & $60(47)$ & .379 \\
\hline Innominate artery & $113(32)$ & $40(40)$ & $55(43)$ & .052 \\
\hline Right axillary artery & $24(7)$ & $6(6)$ & $15(12)$ & .179 \\
\hline One iliac artery & $72(20)$ & $18(18)$ & $27(21)$ & .851 \\
\hline Both iliac arteries & $63(18)$ & $24(24)$ & $26(20)$ & .337 \\
\hline
\end{tabular}

Values are $\mathrm{n}(\%)$ except where otherwise noted. Bold indicates significant results $(P<.05)$. AR, Aortic regurgitation.

malperfusion (odds ratio, $4.065 ; P<.001$ ) were predictors of in hospital mortality.

\section{Innominate Artery Dissection}

Of the 584 patients with ADA, the dissection extended into the innominate artery in 208 patients in this study population. Of those, axillary cannulation was performed in 40 patients compared with 168 patients with nonaxillary cannulation. Similar rates of dissection-related stroke (axillary: $\mathrm{n}=3[5 \%]$; nonaxillary: $\mathrm{n}=23[7 \%]$; $P=.425$ ), procedure-related stroke (axillary: $\mathrm{n}=2[5 \%]$, nonaxillary: $\mathrm{n}=11(7 \%) ; P=1.000)$, and overall stroke (axillary: $\mathrm{n}=5[13 \%]$, nonaxillary: $\mathrm{n}=34$ [20\%]; $P=.367)$ were observed. The aortic dissection extended into the axillary artery in 6 patients in the axillary group and no stroke was diagnosed in those patients. In-hospital mortality was comparable between the 2 groups (axillary: $\mathrm{n}=4[10 \%]$, nonaxillary: $\mathrm{n}=18[11 \%] ; P=1.000)$.

\section{DISCUSSION}

The main findings of this study can be summarized as follows: (1) central ascending aortic cannulation in patients

TABLE 3. Operative details of the study population after inverse probability weighting

\begin{tabular}{|c|c|c|c|c|}
\hline & \multicolumn{3}{|c|}{ Cannulation group } & \multirow[b]{2}{*}{$P$ value } \\
\hline & Aortic ESS $=346$ & Axillary ESS $=94.68$ & Femoral ESS $=119.7$ & \\
\hline \multicolumn{5}{|l|}{ Operative times } \\
\hline Skin incision time, minutes & $316(264-378)$ & $379(310-460)$ & $323(283-403)$ & $<.001$ \\
\hline Operative non-CPB time, minutes* & $116(88-150)$ & $173(137-218)$ & $116(90-156)$ & $<.001$ \\
\hline $\mathrm{CPB}$, minutes & $198(167-238)$ & $212(176-252)$ & $212(181-254)$ & .022 \\
\hline $\mathrm{CX}$, minutes & $125(103-160)$ & $131(105-173)$ & $148(112-179)$ & .021 \\
\hline HCA time, minutes & $32(25-42)$ & $36(27-49)$ & $35(28-55)$ & .0497 \\
\hline With RCP & $414(74)$ & $151(29)$ & $473(90)$ & $<.001$ \\
\hline With ACP & $100(18)$ & $328(62)$ & $17(3)$ & $<.001$ \\
\hline With both RCP and ACP & $38(7)$ & $48(9)$ & $29(6)$ & .493 \\
\hline \multicolumn{5}{|l|}{ Proximal repair } \\
\hline Aortic valve resuspension $†$ & $466(83)$ & $425(81)$ & $424(81)$ & .810 \\
\hline Aortic root replacement & $72(13)$ & $76(14)$ & $77(15)$ & .870 \\
\hline Wheat procedure $\ddagger$ & $12(2)$ & $22(4)$ & $9(2)$ & .405 \\
\hline V-SARR & $10(2)$ & $4(1)$ & $13(3)$ & .471 \\
\hline \multicolumn{5}{|l|}{ Distal repair } \\
\hline Isolated ascending replacement & $6(1)$ & $5(1)$ & $8(2)$ & .801 \\
\hline Hemiarch replacement & $502(90)$ & $362(69)$ & $481(92)$ & $<.001$ \\
\hline Total arch replacement & $52(9)$ & $161(31)$ & $34(6)$ & $<.001$ \\
\hline
\end{tabular}

Values are n (\%) or median (first quartile-third quartile) except where otherwise noted. Bold indicates significant results $(P<.05)$. ESS, Effective sample size; $C P B$, cardiopulmonary bypass time; $C X$, aortic cross-clamp time; $H C A$, hypothermic circulatory arrest, $R C P$, retrograde cerebral perfusion; $A C P$, antegrade cerebral perfusion; $V$-SARR, valve-sparing aortic root replacement. *Defined as the difference between skin incision time and CPB time. $\dagger$ Aortic valve resuspension includes supracoronary ascending replacement, sinus of Valsalva repair with a Teflon felt "neo-media," and valve resuspension in patients with native aortic valve. $\ddagger$ Wheat procedures includes aortic valve replacement, sinus of Valsalva repair with a Teflon felt "neo-media," and supracoronary ascending replacement. 
TABLE 4. Outcome characteristics of the study population after inverse probability weighting

\begin{tabular}{|c|c|c|c|c|}
\hline & \multicolumn{3}{|c|}{ Cannulation group } & \multirow[b]{2}{*}{$P$ value } \\
\hline & $\overline{\text { Aortic ESS }=346}$ & Axillary ESS $=94.68$ & Femoral ESS $=119.7$ & \\
\hline \multicolumn{5}{|l|}{ Transfusion } \\
\hline PRBC, U & $3(1-6)$ & $4(1-9)$ & $4(2-9)$ & .170 \\
\hline FFP, U & $3(2-5)$ & $4(2-7)$ & $4(1-7)$ & .016 \\
\hline Platelets, U & $2(1-3)$ & $2(1-3)$ & $2(1-3)$ & .532 \\
\hline Transfused volume, L & $2.2(1.0-3.7)$ & $2.7(1.4-4.4)$ & $2.4(1.3-5.5)$ & .064 \\
\hline Tracheotomy & $44(8)$ & $57(11)$ & $35(7)$ & .426 \\
\hline Re-exploration for bleeding & $53(10)$ & $50(10)$ & $80(15)$ & .218 \\
\hline Overall stroke & $74(13)$ & $53(10)$ & $65(12)$ & .701 \\
\hline Dissection-related stroke* & $42(7)$ & $18(3)$ & $25(5)$ & .325 \\
\hline Procedure-related stroke $\dagger$ & $32(6)$ & $35(7)$ & $40(8)$ & .783 \\
\hline Temporary neurological dysfunction & $51(10)$ & $41(8)$ & $90(18)$ & .051 \\
\hline Renal failure & $117(22)$ & $129(26)$ & $77(15)$ & .100 \\
\hline Renal replacement therapy & $78(14)$ & $63(13)$ & $49(9)$ & .446 \\
\hline ICU stay, d & $4(2-7)$ & $4(2-7)$ & $3(1-5)$ & .181 \\
\hline In-hospital stay, d & $11(8-18)$ & $12(8-22)$ & $10(8-15)$ & .197 \\
\hline In-hospital mortality & $56(10)$ & $60(11)$ & $69(13)$ & .680 \\
\hline
\end{tabular}

Values are $\mathrm{n}(\%)$ or median (first quartile-third quartile) except where otherwise noted. Bold indicates significant results $(P<.05)$. ESS, Effective sample size; $P R B C$, packed red blood cells; FFP, fresh frozen plasma; ICU, intensive care unit. ${ }^{*}$ Stroke in patients with clear preoperative neurologic deficit in the absence of hemodynamic compromise. $\dagger$ Stroke in patients without clear preoperative neurologic deficit in the absence of hemodynamic compromise.

with ADA can be used as safely as axillary or femoral cannulation; (2) axillary cannulation is more commonly used for patients who require total arch repair, and does not reflect in a higher incidence of postoperative morbidity or mortality; and (3) cannulation of right axillary arteries in patients with ADA with involvement of the innominate artery does not result in worse clinical outcome and can safely be performed when needed.

The ideal cannulation strategy for ADA is quick to establish, minimizes any organ malperfusion, prevents progression of the dissection flap or aortic rupture, and allows optimal brain protection when HCA is needed. ${ }^{16}$ In

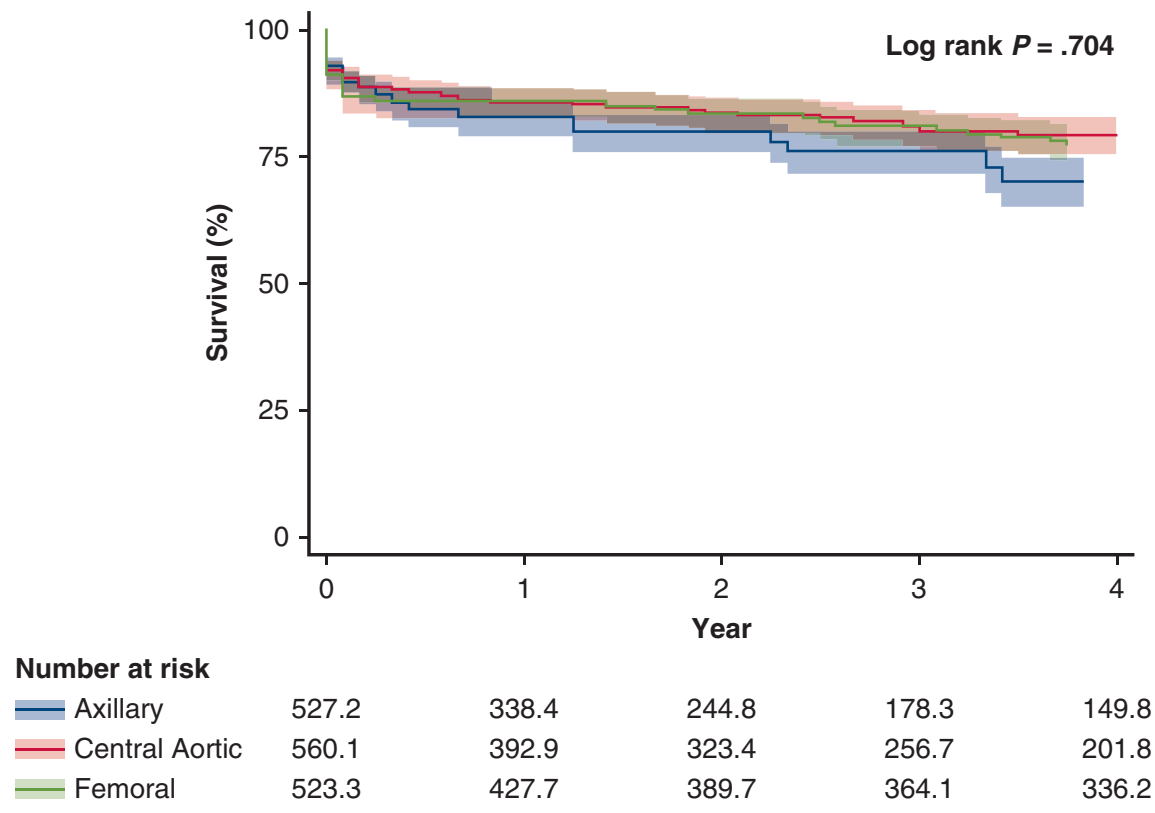

FIGURE 3. Kaplan-Meier analysis survival estimates after surgical repair of acute aortic dissection type A depending on their cannulation site. Ninety-five percent confidence intervals are depicted and effective sample sizes are shown. 
TABLE 5. Logistic regression, in-hospital mortality, weighted*

\begin{tabular}{llc}
\hline \multicolumn{1}{c}{ Variable } & \multicolumn{1}{c}{ OR $(\mathbf{9 5} \% \mathbf{~ C I})$} & $\boldsymbol{P}$ value \\
\hline Male sex & $0.741(0.341-1.611)$ & .450 \\
COPD & $1.451(0.733-2.875)$ & .286 \\
Chronic renal failure & $0.884(0.407-1.917)$ & .755 \\
\hline Age $>80$ y & $5.000(1.319-18.956)$ & $\mathbf{. 0 1 8}$ \\
\hline Shock & $1.451(0.592-3.556)$ & .415 \\
Tamponade & $2.434(0.790-7.499)$ & .121 \\
\hline Any organ malperfusion & $4.065(2.129-7.760)$ & $<. \mathbf{0 0 1}$ \\
\hline Axillary cannulation & $1.464(0.839-2.555)$ & .180 \\
\hline Femoral cannulation & $1.549(0.772-3.107)$ & .218 \\
\hline Bold
\end{tabular}

Bold indicates significant results $(P<.05)$. OR, Odds ratio; $C I$, confidence interval; $C O P D$, chronic obstructive pulmonary disease. *Adjusted for surgeon-specific factors using a mixed effect model.

fact, fast and relatively easy CPB access can usually be accomplished using central aortic or femoral cannulation $^{6,17}$ and shock requiring prompt CPB commencement was more common in patients with those 2 cannulation methods in this study although the difference did not reach statistical significance in the inverse probability weighted analysis. Nevertheless, operative times were shortest in the aortic cannulation group, whereas operative times were significantly longer in the axillary cannulation group. This might be attributed to longer hemostasis times after total arch replacement, but it seems reasonable that the longer time to dissect the axillary artery contributed to the longer operative times as well.

Antegrade perfusion is considered to be advantageous in preventing perioperative malperfusion ${ }^{16,18,19}$ and central aortic and axillary cannulation allow antegrade systemic blood flow, ${ }^{19}$ whereas retrograde perfusion through the femoral arteries might increase the risk for systemic malperfusion because of thrombus embolization and propagation of false lumen perfusion. ${ }^{16,20}$ Moreover, some authors have raised concerns regarding the risk of false lumen cannulation when performing central aortic cannulation. ${ }^{4}$ To avoid this serious complication, it is essential to carefully evaluate the relationship of the true and false lumen in the ascending aorta and aortic arch according to preoperative CT angiography and intraoperative TEE. We strongly recommend using the Seldinger technique and controlling the wire and later the position of the aortic cannula with TEE, which considerably reduces the risk of inserting the cannula into the false lumen. In this study, we did not observe a difference in the cannulation strategy in patients with any preoperative organ malperfusion or with involvement of the supra-aortic vessels. Also, the different perfusion strategies did not result in different rates of perioperative malperfusion as indicated by the similar rates of postoperative stroke, postoperative renal failure, or the need for renal replacement therapy. However, a considerably higher incidence of temporary neurological deficit was observed in the femoral cannulation group $(P=.051)$, and the incidence of procedure-related stroke was the highest in this group too (not significant). Embolization has been reported to be a problem with femoral cannulation. ${ }^{5,7}$ Our data do not prove that this was a perioperative problem, but the possibility of microembolization cannot be ruled out.

Only few centers routinely use central aortic cannulation for the surgical treatment of ADA and the acceptance for central aortic cannulation is limited by concerns regarding its safety. In fact, some authors have reported aortic rupture after central aortic cannulation, ${ }^{4,21}$ but we have not observed one case of aortic rupture after central aortic cannulation in this large case series. We routinely use the Seldinger technique in all cases of central aortic cannulation, which allows controlled insertion of the cannula and minimizes the stress applied to the dissected aortic wall.

The operative details in this study reveal the significant difference among the 3 cannulation groups: axillary cannulation is more commonly used for patients who require total arch repair. This highlights the substantial influence of the location of the dissection tear on the choice of cannulation strategy. In our center, we routinely perform aggressive hemiarch replacement with resection of the entire lesser curvature and most of the dissected aortic arch wall in ADA patients. Total aortic arch replacement is performed when the intimal tear is along the greater curvature of the aortic arch. ${ }^{11}$ Therefore, axillary cannulation is the preferred cannulation method for more complex surgery in patients with more complex aortic dissections. In fact, in the axillary cannulation group HCA times were longest and the incidence of complete aortic dissection was highest. However, central cannulation seems to be the preferred method in patients with proximal ADA and operative times were shortest in this group. Of note, the cannulation method depended on the surgeon's personal preference in this study, and the cannulation method was individualized for each patient. At this point, there is no institutional cannulation policy. Generally, axillary cannulation seems to be the preferred cannulation method when the need for complete arch replacement is anticipated, particularly in stable patients. In unstable patients, central aortic cannulation (especially in patients with tamponade or when complete arch replacement is likely) or femoral cannulation are the preferred strategies.

Despite the significant differences in operative strategy and the invasiveness of the surgery, we observed similar postoperative morbidity among the study groups. In addition, in-hospital mortality was similar among all 3 cannulation groups with a numerically, but not statistically, higher mortality rate in the femoral cannulation group. Late survival analysis revealed no significant statistical difference among the groups (Figure 3), whereas others 
have reported significantly worse long-term survival in patients after central aortic cannulation in smaller collectives. $^{8}$

Lastly, we were able to confirm the safety and efficacy of cannulating the right axillary artery in the setting of a dissected innominate artery for ADA repair. To date, a scarcity of data exist regarding this question and our findings support the previously reported safe usage of the axillary artery cannulation site although the innominate artery is involved in the dissection ${ }^{22}$ when needed. Our cannulation strategy was not influenced by involvement of the innominate artery. However, in cases in which the axillary artery was involved, axillary cannulation was probably not the first choice for arterial inflow, although our data suggest it can be done when no other cannulation method is optimal.

\section{Limitations and Strengths}

Although this was a retrospective single-center study, it is - to the best of our knowledge - the first to compare all 3 commonly used cannulation sites. Also, it is the largest study to compare central aortic cannulation with axillary and femoral cannulation. However, we cannot exclude bias in terms of patient selection and treatment because the cannulation method and the choice of cerebral perfusion remained the individual surgeon's preference. Also, our retrospective database analyses might under-report events and risk factors and clinical outcome analysis focused on postoperative mortality and morbidity, and we were not able to provide information on late complications, quality of life, and cause of death after discharge.

\section{CONCLUSIONS}

Central cannulation in patients with acute ADA can be used as safely as axillary or femoral cannulation, providing another option for quick and easy establishment of CPB. In addition, cannulation of right axillary arteries in patients with ADA with involvement of the innominate artery does not result in worse clinical outcome and can be performed when needed.

\section{Conflict of Interest Statement}

Authors have nothing to disclose with regard to commercial support.

\section{References}

1. Conzelmann LO, Weigang E, Mehlhorn U, Abugameh A, Hoffmann I, Blettner M, et al. Mortality in patients with acute aortic dissection type A: analysis of pre- and intraoperative risk factors from the German Registry for Acute Aortic Dissection Type A (GERAADA). Eur J Cardiothorac Surg. 2015;49:e44-52.

2. Benedetto U, Mohamed H, Vitulli P, Petrou M. Axillary versus femoral arterial cannulation in type A acute aortic dissection: evidence from a meta-analysis of comparative studies and adjusted risk estimates. Eur J Cardiothorac Surg. 2015;48:953-9.

3. Parikh N, Trimarchi S, Gleason TG, Kamman AV, di Eusanio M, Myrmel T, et al. Changes in operative strategy for patients enrolled in the International Registry of Acute Aortic Dissection interventional cohort program. J Thorac Cardiovasc Surg. 2017; 153:S74-9.

4. Reece TB, Tribble CG, Smith RL, Singh RR, Stiles BM, Peeler BB, et al. Central cannulation is safe in acute aortic dissection repair. J Thorac Cardiovasc Surg. 2007;133:428-34.

5. Kamiya H, Kallenbach K, Halmer D, Ozsoz M, Ilg K, Lichtenberg A, et al. Comparison of ascending aorta versus femoral artery cannulation for acute aortic dissection type A. Circulation. 2009;120(11 suppl):S282-6.

6. Frederick JR, Yang E, Trubelja A, Desai ND, Szeto WY, Pochettino A, et al. Ascending aortic cannulation in acute type a dissection repair. Ann Thorac Surg. 2013;95:1808-11.

7. Ren Z, Wang Z, Hu R, Wu H, Deng H, Zhou Z, et al. Which cannulation (axillary cannulation or femoral cannulation) is better for acute type A aortic dissection repair? A meta-analysis of nine clinical studies. Eur J Cardiothorac Surg. 2014;47:408-15.

8. Sabashnikov A, Heinen S, Deppe AC, Zeriouh M, Weymann A, Slottosch I, et al. Axillary or aortic cannulation for aortic repair in patients with stanford A dissection? Ann Thorac Surg. 2016;102:787-94.

9. Rylski B, Bavaria JE, Milewski RK, Vallabhajosyula P, Moser W, Kremens E, et al. Long-term results of neomedia sinus valsalva repair in 489 patients with type A aortic dissection. Ann Thorac Surg. 2014;98:582-8; discussion: 8-9.

10. Bavaria JE, Pochettino A, Brinster DR, Gorman RC, McGarvey ML, Gorman JH, et al. New paradigms and improved results for the surgical treatment of acute type A dissection. Ann Surg. 2001;234:336-42; discussion: 42-3.

11. Rylski B, Milewski RK, Bavaria JE, Vallabhajosyula P, Moser W, Szeto WY, et al. Long-term results of aggressive hemiarch replacement in 534 patients with type A aortic dissection. J Thorac Cardiovasc Surg. 2014;148:2981-5.

12. Bavaria J, Vallabhajosyula P, Moeller P, Szeto W, Desai N, Pochettino A. Hybrid approaches in the treatment of aortic arch aneurysms: postoperative and midterm outcomes. J Thorac Cardiovasc Surg. 2012;145(3 suppl):S85-90.

13. Burgette L, Griffin BA, McCaffrey D. Propensity scores for multiple treatments: a tutorial for the mnps function in the twang package. Available at: https://cran. r-project.org/web/packages/twang/vignettes/mnps.pdf. Accessed July 1, 2018.

14. Ridgeway G, McCaffrey D, Morral A, Burgette L, Griffin BA. Toolkit for Weighting and Analysis of Nonequivalent Groups: a tutorial for the R TWANG package. Available at: https://rand.org/pubs/tools/TL136z1.html. Accessed July $1,2018$.

15. Cole SR, Hernan MA. Constructing inverse probability weights for marginal structural models. Am J Epidemiol. 2008;168:656-64.

16. Abe T, Usui A. The cannulation strategy in surgery for acute type A dissection. Gen Thorac Cardiovasc Surg. 2016;65:1-9.

17. Fusco DS, Shaw RK, Tranquilli M, Kopf GS, Elefteriades JA. Femoral cannulation is safe for type A dissection repair. Ann Thorac Surg. 2004;78: 1285-9; discussion: 9 .

18. El-Hamamsy I, Ouzounian M, Demers P, McClure S, Hassan A, Dagenais F, et al. State of the art surgical management of acute type A aortic dissection. Can J Cardiol. 2016;32:100-9.

19. Peterss S, Pichlmaier M, Curtis A, Luehr M, Born F, Hagl C. Patient management in aortic arch surgery. Eur J Cardiothorac Surg. 2017;51(suppl 1): i4-14.

20. David TE, Armstrong S, Ivanov J, Barnard S. Surgery for acute type A aortic dissection. Ann Thorac Surg. 1999;67:1999-2001; discussion: 14-9.

21. Khaladj N, Shrestha M, Peterss S, Strueber M, Karck M, Pichlmaier M, et al. Ascending aortic cannulation in acute aortic dissection type A: the Hannover experience. Eur J Cardiothorac Surg. 2008;34:792-6. disussion: 6.

22. Rylski B, Czerny M, Beyersdorf F, Kari FA, Siepe M, Adachi H, et al. Is right axillary artery cannulation safe in type A aortic dissection with involvement of the innominate artery? J Thorac Cardiovasc Surg. 2016;152:801-7.e1.

Key Words: type A aortic dissection, cannulation, central cannulation, ascending aorta 


\section{APPENDIX E1. METHODS}

Propensity score pair-matching is widely adopted for causal inference in observational studies via creating matched pairs with similar probability of receiving the same treatment, yet received different treatments. However, patients might be excluded from the study because of lack of appropriate match(es), which will not only decrease the sample size, but also limit the generalizability of the analysis. We aimed to investigate the effect of the cannulation method on all patients who received ADA repair (average treatment effect) with 584 observations. Therefore, inverse probability weighting was adopted to balance baseline characteristics (age, sex, hyperlipidemia, hypertension, diabetes, stroke, chronic renal failure, chronic obstructive pulmonary disease, coronary artery disease, bicuspid aortic valve disease, and Marfan syndrome, shock, tamponade, moderate to severe aortic regurgitation, organ malperfusion, dissection type [limited proximal, innominate, right axillary artery, left subclavian/left common carotid artery, 1 iliac artery, both iliac arteries]). We used the twang package in $\mathrm{R}$ 3.5.1 ( $\mathrm{R}$ Institute for Statistical Computing) to perform matching between 3 cannulation groups with 3000 iterations. Default stopping rules were used (ie, using the absolute standardized bias metrics and summarizes across variables with the mean. The assigned iteration number was determined to be adequate and appropriate; Figure E1). The generated multinomial propensity scores met the overlap assumption, and are graphed in Figure E2. The weights obtained are graphed in Figure E3. After weighing, the standardized mean differences are presented in Love plots (Figures E4 and E5), whereas variables with $>20 \%$ standardized difference are reported in Table E1.

Stabilized weights were calculated by trimming the weights at the 90th percentile (ie, replacing weights over the 90th percentile as the 90th percentile weights). The distributions of stabilized weights are graphed in Figure E5. The results of logistic regression assessing differences of in-hospital mortality with stabilized weights were similar to results from using raw weights (Table E4). The results of Cox regression assessing differences in long-term survival with stabilized weights were also similar to results from using raw weights (Figure E6). 


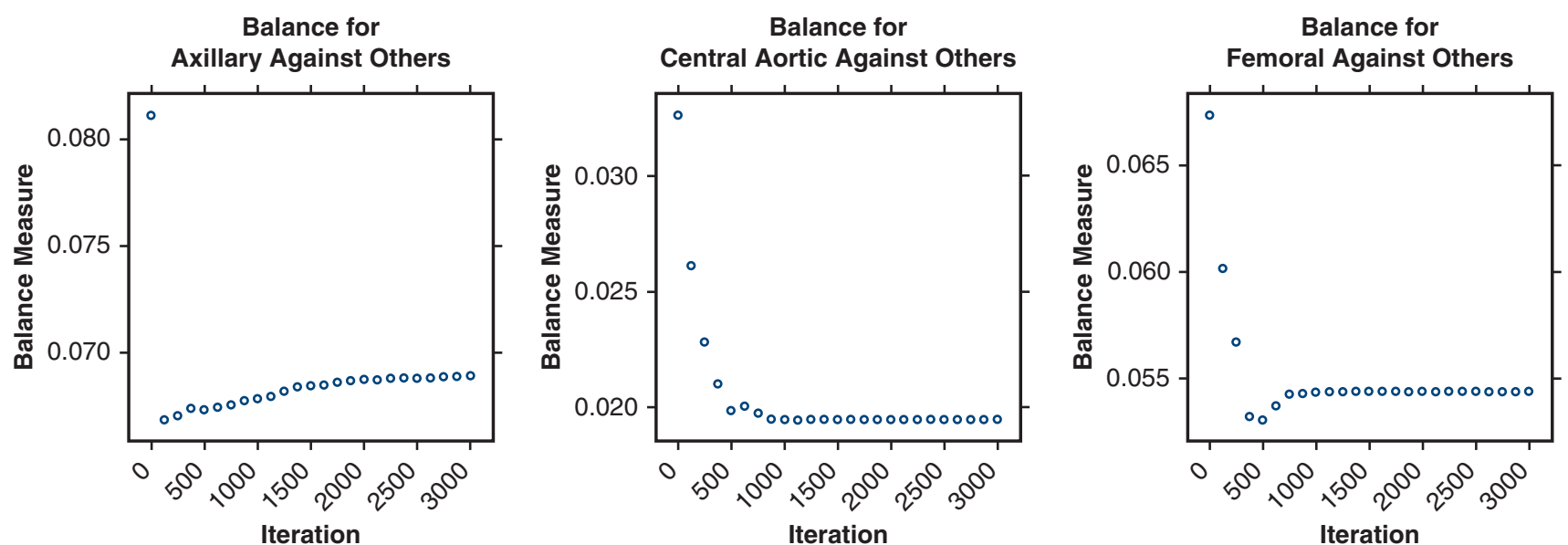

FIGURE E1. Assessment of balance measure over iteration, stratified according to cannulation type. Stabilized balance measure for all 3 groups indicated sufficient iteration to achieve balance among cannulation types.
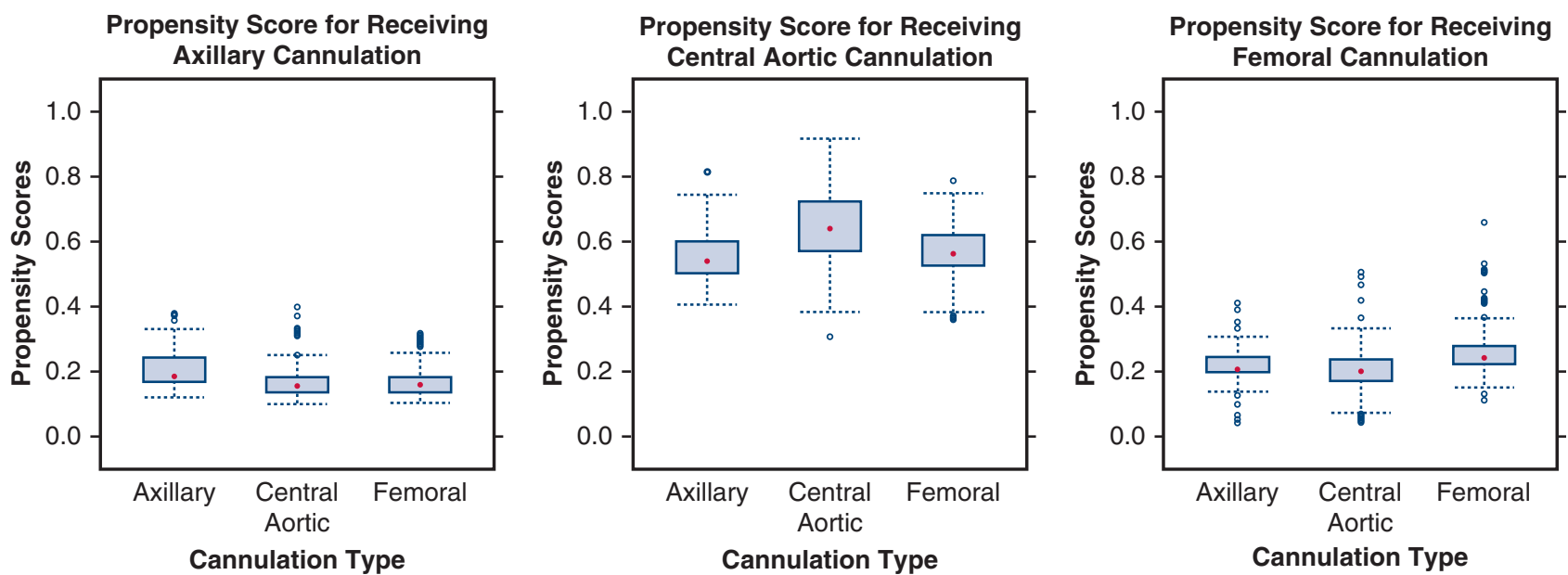

FIGURE E2. Distribution of multinomial propensity scores for different cannulation methods, stratified according to cannulation group. The lower and upper boundaries of the central boxes represent the 25th and 75th percentiles, respectively. The lower and upper whiskers represent the upper and lower adjacent values (25th percentile -1.5 interquartile range and 75 th percentile +1.5 interquartile range), respectively. Red dots represent median; blue dots represent values beyond upper and lower adjacent values. 


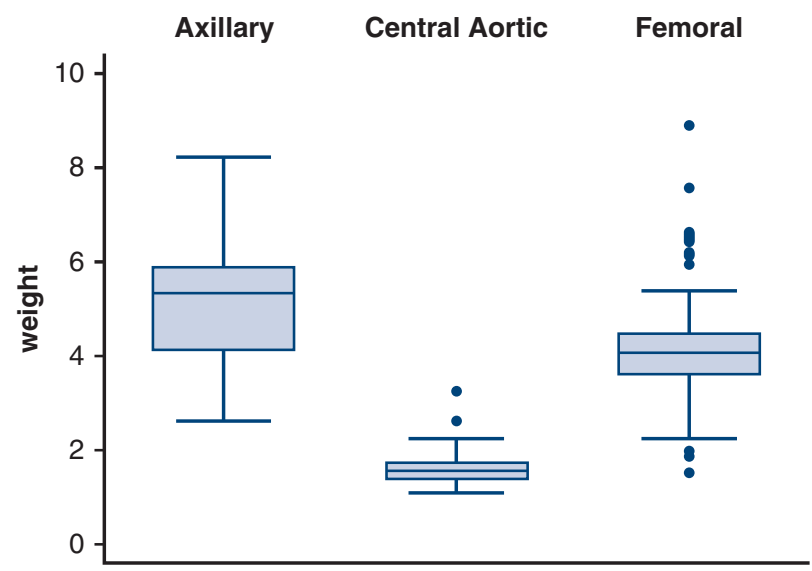

FIGURE E3. Distribution of inverse probability weights for axillary, central aortic, and femoral cannulation. The lower and upper boundary of the central boxes represent the 25 th and 75 th percentiles, respectively. The lower and upper whiskers represent the upper and lower adjacent values (25th percentile -1.5 interquartile range [IQR] and 75 th percentile +1.5 IQR), respectively. Lines in central boxes represent medians. Dots represent values beyond upper and lower adjacent values. Axillary cannulation: 2.63-8.23; mean, 5.22; standard deviation (SD), 1.35; IQR, 4.12-5.91. Central aortic cannulation: 1.09-3.24; mean, 1.58; SD, 0.25; IQR, 1.38-1.75. Femoral cannulation: 1.51-8.90; mean, 4.09; SD, 1.08; IQR, 1.98-8.53. 


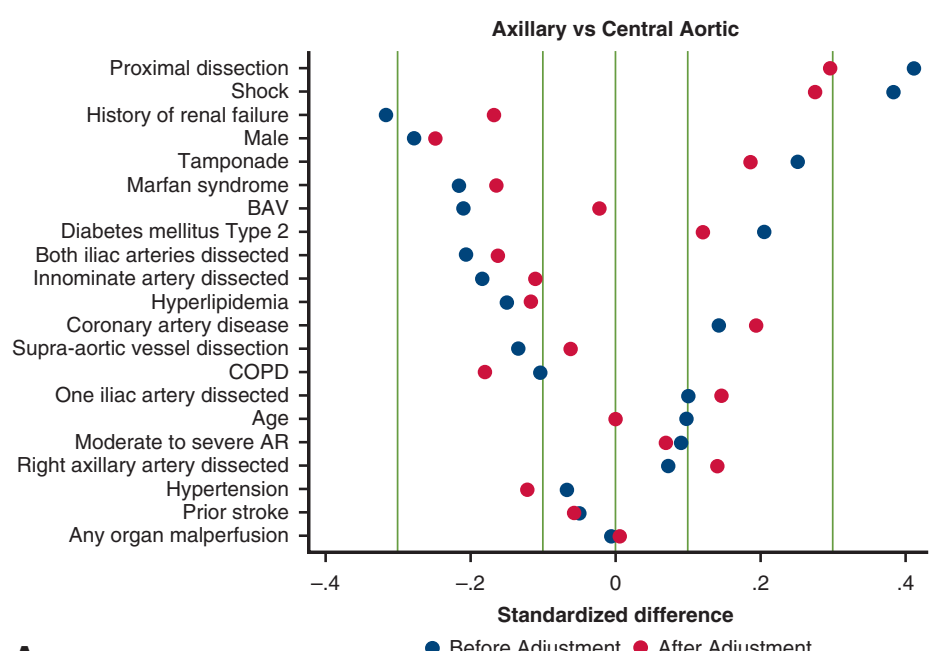

A

- Before Adjustment After Adjustment

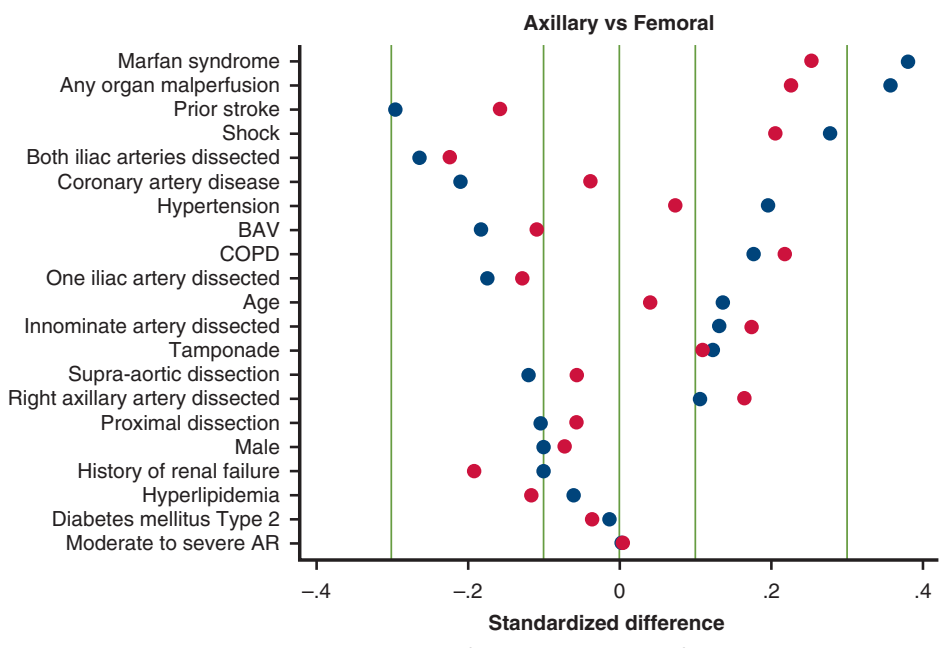

B

- Before Adjustment $\quad$ After Adjustment

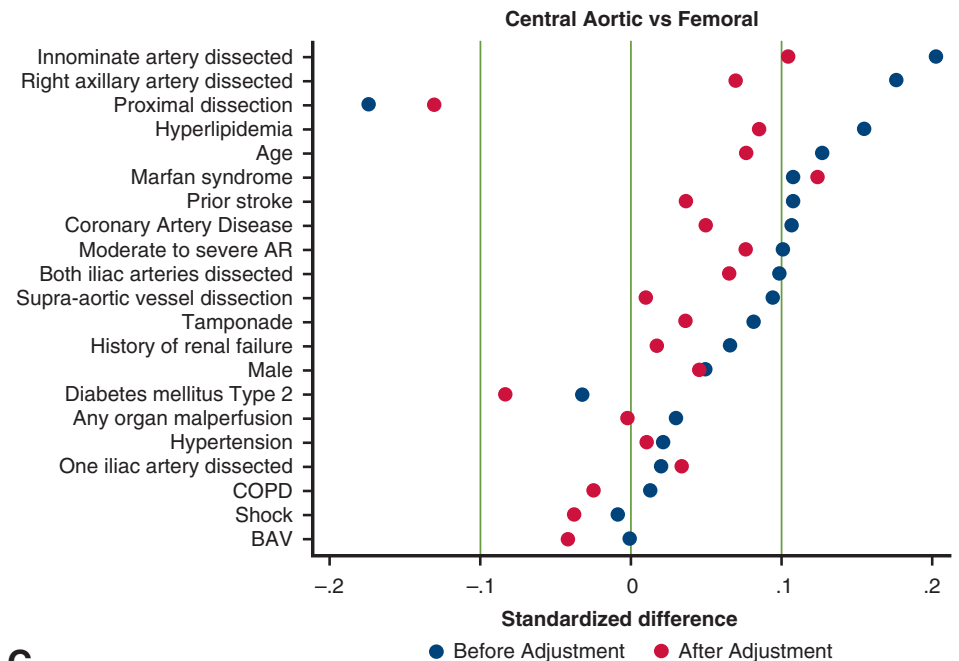

FIGURE E4. Standardized mean differences: (A) axillary versus central aortic; (B) axillary versus femoral; and (C) central aortic versus femoral. $B A V$, Bicuspid aortic valve; $C O P D$, chronic obstructive pulmonary disease; $A R$, aortic regurgitation. 


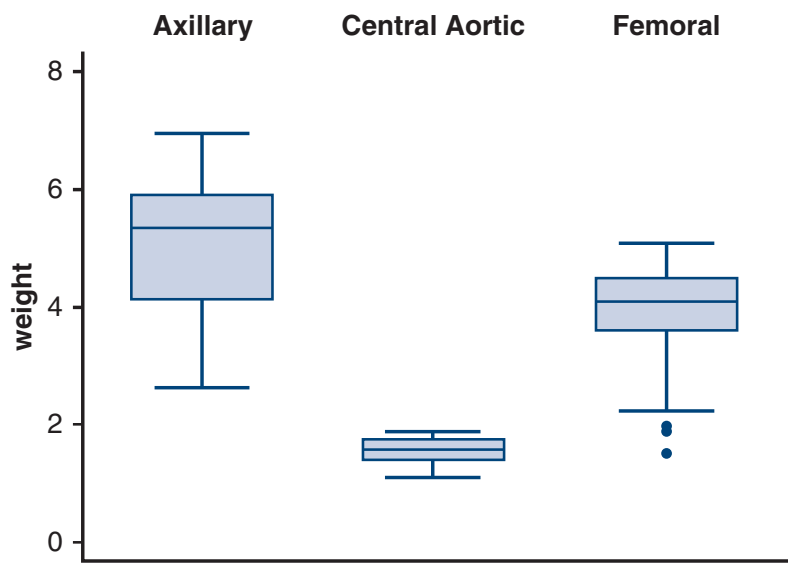

FIGURE E5. Distribution of stabilized inverse probability weights for axillary, central aortic, and femoral cannulation. The lower and upper boundary of the central boxes represent 25th and 75th percentiles, respectively. The lower and upper whiskers represent the lower and upper adjacent values (25th percentile -1.5 interquartile range [IQR] and 75th percentile $+1.5 \mathrm{IQR}$ ), respectively. Lines in central boxes represent medians. Dots represent values beyond upper and lower adjacent values. Axillary cannulation: 2.63-6.96; mean, 5.16; standard deviation (SD), 1.27; IQR, 4.12-5.91. Central aortic cannulation: 1.09-1.87; mean, 1.56; SD, 0.22; IQR, 1.38-1.75. Femoral cannulation: 1.51-5.08; mean, 3.98; SD, 0.83; IQR, 1.98-4.51.

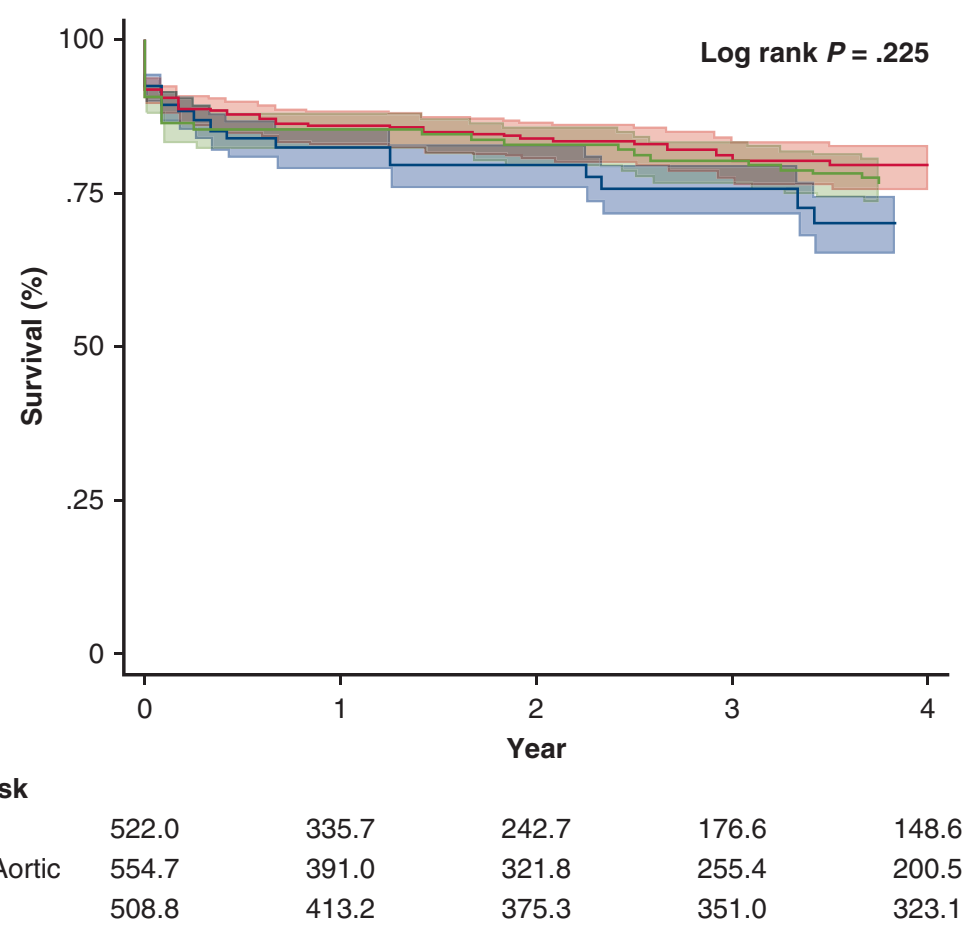

FIGURE E6. Kaplan-Meier analysis survival estimates after surgical repair of acute aortic dissection type A depending on their cannulation site using stabilized weights; $95 \%$ confidence intervals are depicted and effective sample sizes are shown. 
TABLE E1. Standardized mean difference decrease in variables with $>\mathbf{2 0} \%$ standardized difference

\begin{tabular}{lccc}
\hline & \multicolumn{2}{c}{ Standardized difference } & \\
\cline { 2 - 3 } & $\begin{array}{c}\text { Before } \\
\text { weighting }\end{array}$ & $\begin{array}{c}\text { After } \\
\text { weighting }\end{array}$ & Decrease, \% \\
\hline Central aortic vs axillary & & & \\
$\quad$ Sex & -0.274 & -0.251 & 8.39 \\
Shock & 0.402 & 0.294 & 26.87 \\
Limited proximal & 0.403 & 0.286 & 29.03 \\
$\quad$ & & & \\
$\quad$ aortic dissection & & & \\
Femoral vs axillary & 0.250 & 0.234 & 6.40 \\
Coronary artery disease & 0.378 & 0.236 & 37.57 \\
$\quad$ Shock & & & \\
\hline
\end{tabular}

TABLE E2. Baseline characteristics of the study population after inverse probability weighting

\begin{tabular}{|c|c|c|c|c|}
\hline & \multicolumn{3}{|c|}{ Cannulation group } & \multirow[b]{2}{*}{$P$ value } \\
\hline & Aortic ESS $=346$ & Axillary ESS $=94.68$ & Femoral ESS $=119.7$ & \\
\hline Age, $y$ & $60 \pm 18$ & $59 \pm 10$ & $60 \pm 12$ & .999 \\
\hline Male sex & $355(63)$ & $360(68)$ & $343(66)$ & .679 \\
\hline Hyperlipidemia & $222(40)$ & $248(47)$ & $209(40)$ & .359 \\
\hline Hypertension & $494(88)$ & $479(91)$ & $456(87)$ & .605 \\
\hline Diabetes mellitus type 2 & $71(13)$ & $73(14)$ & $54(10)$ & .652 \\
\hline Previous stroke & $52(9)$ & $51(10)$ & $50(10)$ & .980 \\
\hline Chronic renal failure & $58(10)$ & $66(13)$ & $63(12)$ & .808 \\
\hline COPD & $71(13)$ & $78(15)$ & $61(12)$ & .651 \\
\hline Coronary artery disease & $56(10)$ & $22(4)$ & $54(10)$ & .077 \\
\hline Bicuspid aortic valve & $18(3)$ & $27(5)$ & $16(3)$ & .521 \\
\hline Marfan syndrome & $8(1)$ & $22(4)$ & $13(3)$ & .335 \\
\hline
\end{tabular}

Values are $\mathrm{n}(\%)$ or mean \pm standard deviation except where otherwise noted. ESS, Effective sample size; COPD, chronic obstructive pulmonary disease.

TABLE E3. Operative characteristics of the study population after inverse probability weighting

\begin{tabular}{|c|c|c|c|c|}
\hline & \multicolumn{3}{|c|}{ Cannulation group } & \multirow[b]{2}{*}{$P$ value } \\
\hline & Aortic ESS = 346 & Axillary ESS $=94.68$ & Femoral ESS $=119.7$ & \\
\hline Shock & $161(29)$ & $114(22)$ & $151(29)$ & .356 \\
\hline Tamponade & $122(22)$ & $113(22)$ & $125(24)$ & .889 \\
\hline Moderate to severe AR & $181(32)$ & $139(26)$ & $190(36)$ & .254 \\
\hline Any organ malperfusion & 214 (38) & $170(32)$ & $205(39)$ & .426 \\
\hline \multicolumn{5}{|l|}{ Dissection extension } \\
\hline Limited, proximal dissection & $157(28)$ & 98 (19) & $127(24)$ & .204 \\
\hline Supra-aortic vessel dissection & $236(42)$ & $227(43)$ & $237(45)$ & .810 \\
\hline Innominate artery & $188(34)$ & $197(37)$ & $215(41)$ & .374 \\
\hline Right axillary artery & $42(8)$ & $37(7)$ & $48(9)$ & .739 \\
\hline One iliac artery & $114(20)$ & 84 (16) & $112(21)$ & .457 \\
\hline Both iliac arteries & $103(18)$ & $121(23)$ & $104(20)$ & .525 \\
\hline
\end{tabular}

Values are $\mathrm{n}(\%)$ except where otherwise noted. ESS, Effective sample size; $A R$, aortic regurgitation.

\section{4.e6 The Journal of Thoracic and Cardiovascular Surgery • July 2019}


TABLE E4. Logistic regression, in-hospital mortality, using stabilized weight*

\begin{tabular}{lcc}
\hline \multicolumn{1}{c}{ Variable } & OR $(\mathbf{9 5} \% \mathbf{C I})$ & $\boldsymbol{P}$ value \\
\hline Male sex & $0.800(0.339-1.888)$ & .610 \\
COPD & $1.428(0.702-2.904)$ & .326 \\
\hline Chronic renal failure & $0.890(0.417-1.900)$ & .763 \\
Age $>80$ y & $5.421(1.347-21.82)$ & $\mathbf{. 0 1 7}$ \\
\hline Shock & $1.552(0.650-3.708)$ & .322 \\
Tamponade & $2.308(0.790-6.743)$ & .126 \\
Any organ malperfusion & $\mathbf{3 . 9 3 5}(2.084-7.429)$ & $<.001$ \\
\hline Axillary cannulation & $1.493(0.806-2.766)$ & .203 \\
\hline $\begin{array}{l}\text { Femoral cannulation } \\
\text { Bold indicates significant results }(P<.05) . \text { OR, Odds ratio; } C I, \text { confidence interval; } \\
\text { COPD, chronic obstructive pulmonary }\end{array}$ disease. ${ }^{*}$ Adjusted for surgeon-specific \\
factors using a mixed effect model.
\end{tabular}


TABLE E5. Balance of covariates between cannulation types before and after inverse probability weighting

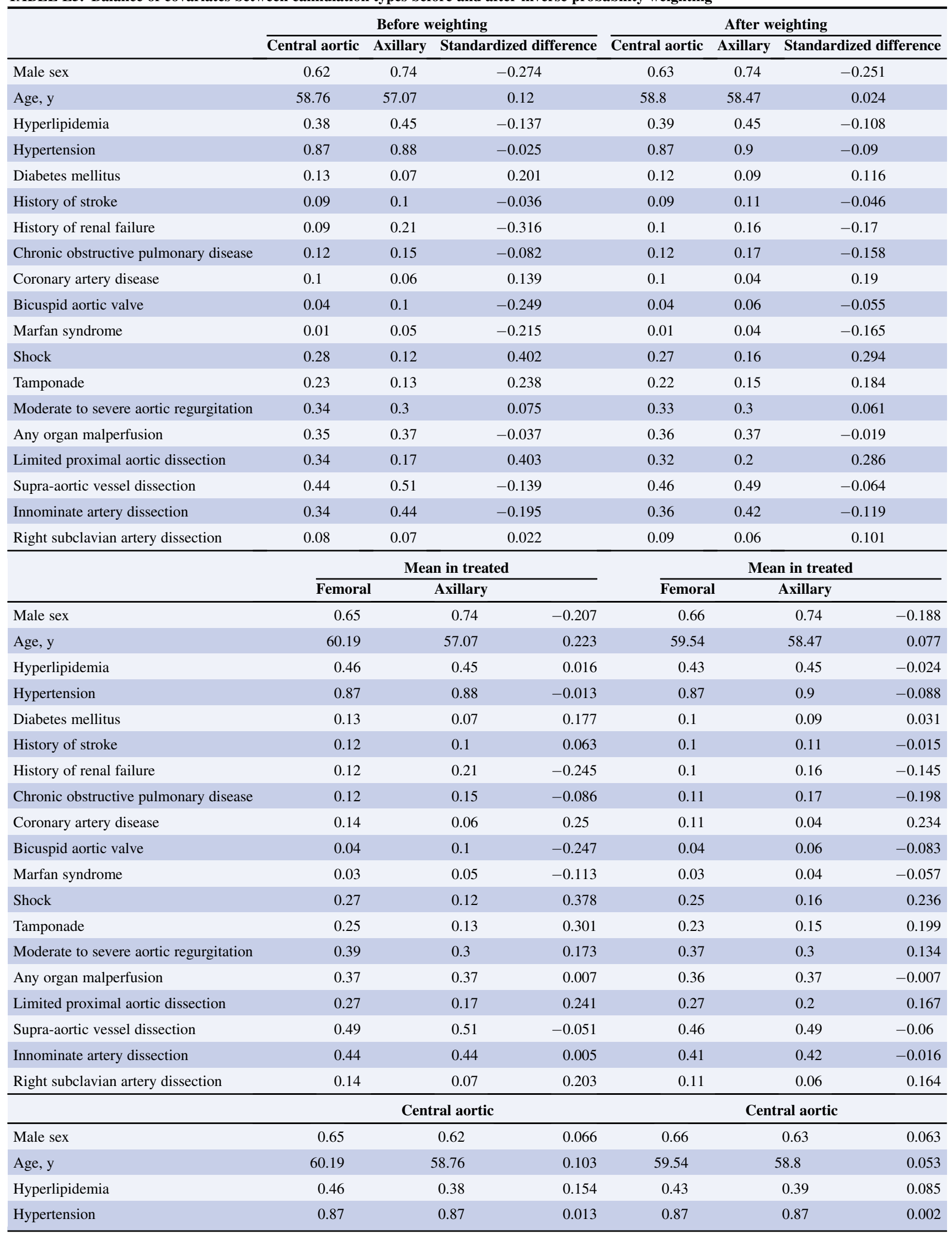


TABLE E5. Continued

\begin{tabular}{|c|c|c|c|c|c|c|}
\hline \multirow[b]{2}{*}{ Diabetes mellitus } & \multicolumn{3}{|c|}{ Central aortic } & \multicolumn{3}{|c|}{ Central aortic } \\
\hline & 0.13 & 0.13 & -0.024 & 0.1 & 0.12 & -0.076 \\
\hline History of stroke & 0.12 & 0.09 & 0.099 & 0.1 & 0.09 & 0.029 \\
\hline History of renal failure & 0.12 & 0.09 & 0.072 & 0.1 & 0.1 & 0.023 \\
\hline Chronic obstructive pulmonary disease & 0.12 & 0.12 & -0.005 & 0.11 & 0.12 & -0.042 \\
\hline Coronary artery disease & 0.14 & 0.1 & 0.113 & 0.11 & 0.1 & 0.056 \\
\hline Bicuspid aortic valve & 0.04 & 0.04 & 0.002 & 0.04 & 0.04 & -0.038 \\
\hline Marfan syndrome & 0.03 & 0.01 & 0.11 & 0.03 & 0.01 & 0.126 \\
\hline Shock & 0.27 & 0.28 & -0.023 & 0.25 & 0.27 & -0.052 \\
\hline Tamponade & 0.25 & 0.23 & 0.063 & 0.23 & 0.22 & 0.018 \\
\hline Moderate to severe aortic regurgitation & 0.39 & 0.34 & 0.098 & 0.37 & 0.33 & 0.073 \\
\hline Any organ malperfusion & 0.37 & 0.35 & 0.045 & 0.36 & 0.36 & 0.012 \\
\hline Limited proximal aortic dissection & 0.27 & 0.34 & -0.16 & 0.27 & 0.32 & -0.117 \\
\hline Supra-aortic vessel dissection & 0.49 & 0.44 & 0.088 & 0.46 & 0.46 & 0.004 \\
\hline Innominate artery dissection & 0.44 & 0.34 & 0.2 & 0.41 & 0.36 & 0.103 \\
\hline Right subclavian artery dissection & 0.14 & 0.08 & 0.181 & 0.11 & 0.09 & 0.075 \\
\hline
\end{tabular}

